\title{
Moderating Effect of Gender in the Relationship between Depression and Waketime Procrastination in Insomnia Patients
}

\author{
Jia Kim, BA*, Sooyeon Suh, PhD* \\ Department of Psychology, Sungshin Women's University, Seoul, Korea
}

\begin{abstract}
Background and Objective Waketime procrastination (WP) is a behavior involving delaying getting out of bed in the morning despite being awake. Previous studies have noted a relationship between WP and depression, but there have been a lack of empirical studies investigating this relationship. This study aimed to examine the moderating effect of gender in the relationship between depression and WP in insomnia patients.
\end{abstract}

Methods Participants consisted of 78 adults $($ men $=52.6 \%$, women $=47.4 \%$, who met DSM- 5 diagnostic criteria for insomnia disorder. All participants completed the Korean version of Center for Epidemiologic Studies Depression Scale-Revised (K-CESD-R) and sleep diaries for a week. WP behavior was calculated based on sleep diary data using the difference in time between wake time and time out of bed. Weekday and weekend sleep diary data were analyzed separately, as many individuals display different sleep behaviors on the weekend compared to the weekdays.

Results Results indicated a significant positive relationship between WP and depression ( $\mathrm{r}=$ $0.335, \mathrm{p}<0.01)$. Gender moderated the relationship between depression and WP on both weekdays and weekends $[\mathrm{B}=-1.239,95 \%$ confidence interval $(\mathrm{CI})=-2.092,-0.386$ and $\mathrm{B}=2.136,95 \% \mathrm{CI}=$ $0.643,3.630$, respectively]. Men with high depression showed more WP on weekdays, while women with high depression showed more WP on weekends.

Conclusions WP may be an important behavior to consider in the clinical context of providing sleep treatment for individuals who are depressed, especially when considering gender differences.

Sleep Med Res 2019;10(2):90-96

\footnotetext{
Received: November 26, 2019

Revised: December 23, 2019

Accepted: December 24, 2019

Correspondence

Sooyeon Suh, $\mathrm{PhD}$

Department of Psychology,

Sungshin Women's University,

2 Bomun-ro 34da-gil, Seongbuk-gu,

Seoul 02844, Korea

Tel $+82-2-920-7215$

Fax +82-2-920-7215

E-mail alysuh@sungshin.ac.kr

*These authors contributed equally to this work.

ORCID

Jia Kim

https://orcid.org/0000-0003-3913-405X

Sooyeon Suh

https://orcid.org/0000-0003-0644-8634
}

Key Words Waketime, Procrastination, Depression, Insomnia, Gender.

\section{INTRODUCTION}

Waketime procrastination (WP) can be defined as the delay in getting out of bed in the morning after waking up. Previous studies have identified this as a problematic form of sleep behavior, particularly with regards to insomnia. For instance, it is common for individuals with insomnia to stay in bed longer than those without insomnia in the hopes of catching more sleep [1]. Most studies with insomnia patients have only focused on reducing sleep onset latency (SOL) and wake after sleep onset (WASO) [2], but relatively little attention has been given to WP.

Insomnia patients often experience early morning awakenings, and may show WP because they may try to stay in bed longer in the morning to make up for insufficient sleep. However, there is not enough research on WP from the perspective of procrastination and related psychological variables such as depression.

Insomnia and depression are highly comorbid, with about $40 \%$ of depressed patients reporting insomnia symptoms [3,4]. One common pattern of behavior observed in both of these two conditions is long periods of time spent in bed $[1,5,6]$. Previous studies have suggested that WP is associated with depression, as depressed people having a tendency to use the bed as an escape to solve affective problems, and stay in bed longer than less depressed people [5-7]. As such, WP 
is a useful behavior that requires further study in the context of depression. However, there has been a lack of direct empirical studies investigating the relationship between WP and depression.

Previous studies have indicated that it is necessary to explore sleep behaviors on weekdays and weekends separately because it is common for insomnia patients to exhibit different sleeping patterns on weekdays vs. weekends [8] which may be reflected in WP. In particular, WP may be more pronounced on weekends, when an individual may have more time to stay in bed and attempt to compensate for sleep loss without having negative consequences on their work or school.

Furthermore, gender differences in insomnia and depression patients are common [9-12]. Problematic sleep patterns associated with insomnia are typically more pronounced in women compared to men [12]. According to previous studies, women have longer SOL and WASO than men, which implies that women may be more likely to spend more time in bed (TIB) awake compared to men [13]. Additionally, it is common for gender differences to be observed in terms of coping behavior related to depression [9]. Men tend to prefer avoidant coping such as physical activity to relieve depression, while women tend to ruminate on the causes and implications of their depression [14]. This suggests that women may be more likely to cope with their depression (e.g., ruminate) in bed compared to men, hence displaying increased WP. These observations suggest that WP associated with insomnia and depression may be more pronounced in women.

Thus, the current study aims to investigate the relationship between WP, depression, and sleep in insomnia patients while considering weekend and weekday differences. We aimed to investigate the moderating effect of gender on the relationship between depression and WP according to weekdays and weekends.

\section{METHODS}

\section{Participants and Procedures}

Participants consisted of 78 adults $($ men $=52.6 \%$, women $=$ $47.4 \%$ ), ranging in age from 20 to 49 years old. All participants were insomnia patients who met DSM-5 diagnostic criteria. Exclusion criteria were individuals with 1) history of psychosis, 2) suicide attempts, 3) shift workers, and 4) individuals who were currently receiving pharmacotherapy or psychotherapy for insomnia. The subjects were recruited through online advertisements in the community. All participants underwent phone screening and were scheduled for an in-person laboratory visit if eligible. During the laboratory visit, all participants completed questionnaires, and received education about completing sleep diaries. Data for sleep diaries were collected online for 7 days. This study was approved by the Institutional Review Board ethics committee (IRB number: SSWUIRB 2017-073).

\section{Measures}

\section{Demographic information}

Demographic information was collected from participants, including their gender and age.

\section{Sleep diary}

Participants completed a daily sleep diary for 7 days. Sleep diaries provide daily subjective estimate parameters about sleep, including bed time, SOL, frequency of nocturnal awakenings, WASO, wake time (WT), time out of bed, feeling upon arising and sleep quality. WP was derived from the sleep diary, and was operationally defined as the time spent from WT to time out of bed. TIB (the length of time spent in bed), total sleep time (TST; total time spent asleep), and sleep efficiency (SE; ratio of TST to TIB multiplied by $100 \%$ ) were also calculated based on sleep diary parameters.

\section{Korean version of Center for Epidemiologic Studies Depression Scale Revised}

The CESD scale was developed to measure severity of depression symptom [15]. The Korean version of Center for Epidemiologic Studies Depression Scale Revised (K-CESD-R) is a 20 -item self-report questionnaire that evaluates the severity of depression symptom [16]. Subjects respond to the degree they have experienced depression symptoms in the past week and how often they experience depression symptoms during the week. Each of the KCESD-R items are scored on a 5-point Likert scale ranging from $0-4$. Total scores range from 0 to 80 , with higher scores reflecting higher symptomatology [17]. According to Van Dam and Earleywine [18], a cut off score of 16 is recommended to determine clinical levels of depression. Cronbach's $\alpha$ of the K-CESD-R in this sample was 0.914 .

\section{Insomnia Severity Index}

Insomnia Severity Index (ISI) is a 7 item self-report questionnaire that evaluates the severity of insomnia, each of items include factors that difficulty falling asleep and staying asleep, problems waking up to early, satisfaction with current sleep patterns and difficulty with daily functions etc. Each of ISI items are scored on a 5 -point Likert scale, rating on a scale of 0 to 4 . A total score is calculated by summing up all items, ranging from 0 to 28 . In ISI, the higher scores indicate more severe insomnia [17]. Cronbach's $\alpha$ of the ISI in this sample was 0.640 .

\section{Statistical Analyses}

All statistical analyses were conducted by using SPSS Statistics version 21 (IBM Corp., Armonk, NY, USA). All parameters were calculated separately for weekdays and weekends. Correlational analyses were conducted using Pearson's correlation coefficient between sleep variables and clinical indicators. T-tests were conducted to determine gender differences in WP and other 
variables. Some variables were analyzed with non-parametric analysis because they did not meet normality assumptions (MannWhitney test). Moderation analysis was conducted by using SPSS Process Macro (A Division of Guilford Publications Inc., New York, NY, USA). We examined the moderating effect of gender on the relationship between depression and WP after adjusting for insomnia severity. For verifying data analysis, we used bootstrapping that was proposed by Shrout and Bolger [19]. Using this model, the relationship is interpreted as statistically significant if the $95 \%$ confidence interval of the repeated sample does not include zero.

\section{RESULTS}

\section{Gender Differences in Sleep and Depression}

General characteristics of the sample are shown in Table 1. Table 1 presents gender differences for sleep and clinical variables. Sleep variables are presented separately for weekdays and weekends. Gender differences for WP were significant on weekdays ( $\mathrm{p}$ $=0.003)$ and weekends $(p=0.034)$. Women engaged in more WP than men during both weekdays and weekends. Both women and men engaged in more WP on weekends than on weekdays.

There was a significant gender difference for severity of depression $(\mathrm{p}=0.016)$, with women reporting feeling more depressed than men. There were also gender differences for SOL, TIB, and SE, which was significant for both weekdays [SOL $(p<0.001)$, TIB $(p=0.006), S E(p<0.001)]$ and weekends [SOL $(p=0.039)$, TIB $(p=0.038), S E(p=0.003)]$. Women took longer to fall asleep than men during both weekdays and weekends and spent more TIB, and had lower SE compared to men. The gender difference for WT on weekdays $(\mathrm{p}=0.157)$ and weekends $(\mathrm{p}=0.224)$ was not significant. In our study, 60 people woke up earlier than planned on weekdays, and 48 on weekends.

\section{Association between WP, Sleep, and Depression}

Table 2 presents the correlations between WP, sleep variables (WT, SOL, WASO, TST, TIB, SE), and depression (K-CESD-R). WP was significantly positively correlated with depression scores on both weekdays and weekends ( $\mathrm{ps}<0.01$ ). Thus, higher levels of WP on weekdays and weekends were associated with more depression symptoms.

Table 1. General Characteristics

\begin{tabular}{|c|c|c|c|c|}
\hline & Mean (SD) & $\begin{array}{c}\text { Men, M (SD) } \\
(\mathrm{n}=41)\end{array}$ & $\begin{array}{l}\text { Women, M (SD) } \\
\quad(\mathrm{n}=37)\end{array}$ & $\mathrm{p}$ \\
\hline Age & $31.74(6.76)$ & $32.83(6.83)$ & $30.54(6.56)$ & 0.136 \\
\hline \multicolumn{5}{|c|}{ Clinical indicators } \\
\hline K-CESD-R & $19.44(12.22)$ & $16.29(10.15)$ & $22.92(13.47)$ & $0.016^{*}$ \\
\hline ISI & $17.97(4.00)$ & $17.63(3.74)$ & $18.35(4.20)$ & 0.905 \\
\hline \multicolumn{5}{|c|}{ Sleep variables (weekdays) } \\
\hline WP & $23.17(23.48)$ & $18.22(24.00)$ & $28.66(21.93)$ & $0.003^{* *}$ \\
\hline SOL & $55.70(39.66)$ & $37.01(24.41)$ & $76.42(43.17)$ & $<0.001^{* * *}$ \\
\hline WASO & $29.68(22.39)$ & $25.98(18.48)$ & $33.79(25.70)$ & 0.132 \\
\hline WT & AM 07:30 (1 h 29 m) & AM 7:12 (1 h 14 m) & AM 7:51 (1 h 40 m) & 0.157 \\
\hline TST & 349.87 (69.29) & $351.81(68.29)$ & $347.72(71.26)$ & 0.796 \\
\hline TIB & $458.43(83.50)$ & $433.02(69.62)$ & $486.58(89.33)$ & $0.006^{* *}$ \\
\hline SE & $76.80(10.44)$ & $81.33(9.24)$ & $71.78(9.44)$ & $<0.001^{* * *}$ \\
\hline \multicolumn{5}{|c|}{ Sleep variables (weekends) } \\
\hline WP & $31.12(45.83)$ & $20.12(16.97)$ & $43.31(62.31)$ & $0.034^{*}$ \\
\hline SOL & $53.20(44.40)$ & $42.52(33.41)$ & $65.03(52.00)$ & $0.039^{*}$ \\
\hline WASO & $29.19(28.96)$ & $26.95(28.90)$ & $31.66(29.20)$ & 0.477 \\
\hline WT & AM 08:10 (1 h $45 \mathrm{~m})$ & $\operatorname{AM} 7: 58(1$ h 39 m) & AM 8:24 (1 h 52 m) & 0.224 \\
\hline TST & 369.72 (87.89) & $375.50(84.82)$ & 363.31 (91.91) & 0.722 \\
\hline TIB & $483.22(105.48)$ & $465.10(92.41)$ & $503.31(116.28)$ & $0.038^{*}$ \\
\hline SE & $77.15(11.76)$ & $80.80(10.35)$ & $73.11(12.04)$ & $0.003^{* *}$ \\
\hline
\end{tabular}

Some variables were analyzed with Mann-Whitney test because they did not meet normality assumptions (weekdays: WP, WT, TIB, SE; weekends: WT, SOL, TST, TIB). ${ }^{*} \mathrm{p}<0.05,{ }^{* *} \mathrm{p}<0.01,{ }^{* * *} \mathrm{p}<0.001$.

K-CESD-R: The Korean version of Center for Epidemiologic Studies Depression Scale Revised, ISI: Insomnia Severity Invetory, WP: waketime procrastination, WT: wake time, SOL: sleep onset latency, WASO: wake after sleep onset, TST: total sleep time, TIB: time in bed, SE: sleep efficiency. 


\section{Moderating Effect of Gender on the Depression-WP Relationship based on Weekdays and Weekends}

Results for the moderator analyses are shown in Table 3. On

Table 2. Association between WP and variables

\begin{tabular}{lc}
\hline \multicolumn{1}{c}{ Variables } & WP correlation \\
\hline Weekdays & $0.335^{* *}$ \\
K-CESD-R & $0.233^{*}$ \\
ISI & -0.013 \\
WT & $0.310^{* *}$ \\
SOL & $0.314^{* *}$ \\
WASO & $-0.269^{*}$ \\
TST & $0.290^{*}$ \\
TIB & \\
Weekends & $0.542^{* *}$ \\
K-CESD-R & $0.292^{* *}$ \\
ISI & -0.219 \\
WT & $0.268^{*}$ \\
TIB & $-0.621^{* *}$ \\
SE & \\
\hline
\end{tabular}

${ }^{*} \mathrm{p}<0.05,{ }^{* *} \mathrm{p}<0.01$.

WP: waketime procrastination, K-CESD-R: The Korean version of Center for Epidemiologic Studies Depression Scale Revised, ISI: Insomnia Severity Invetory, WT: wake time, SOL: sleep onset latency, WASO: wake after sleep onset, TST: total sleep time, TIB: time in bed, SE: sleep efficiency. weekdays, results indicated that there was a significant main effect for depression $(B=1.298, p<0.001)$ and gender $(B=30.108$, $\mathrm{p}=0.002)$. Gender had a moderating effect on the relationship between depression and WP $(\mathrm{B}=-1.239, \mathrm{p}=0.005)$. Meanwhile, on weekends, the main effect for depression $(B=0.478, p=0.466)$ and gender $(B=-29.456, p=0.079)$ were not significant. However, there was a significant moderating effect of gender on the relationship between depression and WP $(B=2.136, \mathrm{p}=0.006)$.

To test the effect size of the moderating variables, we confirmed that gender was a moderator using bootstrapping. Through confirming to effect of depression severity on WP depending on gender differences, we tested the significance of the interaction effect (Table 3). On weekdays, the moderating effect was significant for men $(B=1.298, S E=0.373, t=3.481, p<0.001)$, but not for women $(B=0.059, S E=0.283, t=0.209, p=0.835)$. On weekends, meanwhile, the moderating effect was not significant for men $(B=0.478, S E=0.653, t=0.732, p=0.466)$, but significant for women $(B=2.615, S E=0.495, t=5.280, p<0.001)$. These results suggest that for men, the more depressed they were on weekdays, the more they engaged in WP. In contrast, on weekends, the more depressed that women felt, the more they engaged in WP. These results are presented in Figs. 1 and 2.

\section{DISCUSSION}

The main finding of this study was that WP was related to depression and that individuals with high levels of depression en-

Table 3. The effect of depression on WP: the moderating effects of gender

\begin{tabular}{|c|c|c|c|c|c|c|}
\hline & B & SE & $\mathrm{t}$ & $\mathrm{p}$ & LLCI & ULCI \\
\hline \multicolumn{7}{|l|}{ Weekdays } \\
\hline Constant & -5.976 & 11.944 & -0.500 & 0.618 & -29.781 & 17.829 \\
\hline Depression (A) & 1.298 & 0.373 & 3.481 & 0.001 & 0.555 & 2.041 \\
\hline Gender (B) & 30.108 & 9.452 & 3.186 & 0.002 & 11.271 & 48.945 \\
\hline Interaction $\left(\mathrm{A}^{*} \mathrm{~B}\right)$ & -1.239 & 0.428 & -2.896 & 0.005 & -2.092 & -0.386 \\
\hline \multicolumn{7}{|l|}{ Weekends } \\
\hline Constant & -0.407 & 20.925 & -0.019 & 0.985 & -42.110 & 41.296 \\
\hline Depression (A) & 0.478 & 0.653 & 0.732 & 0.466 & -0.824 & 1.781 \\
\hline Gender (B) & -29.456 & 16.558 & -1.779 & 0.079 & -62.455 & 3.544 \\
\hline Interaction $\left(\mathrm{A}^{*} \mathrm{~B}\right)$ & 2.136 & 0.749 & 2.850 & 0.006 & 0.643 & 3.630 \\
\hline \multicolumn{7}{|l|}{ Weekdays } \\
\hline Men & 1.298 & 0.373 & 3.481 & 0.001 & 0.555 & 2.041 \\
\hline Women & 0.059 & 0.283 & 0.209 & 0.835 & -0.504 & 0.623 \\
\hline \multicolumn{7}{|l|}{ Weekends } \\
\hline Men & 0.478 & 0.653 & 0.732 & 0.466 & -0.824 & 1.781 \\
\hline Women & 2.615 & 0.495 & 5.280 & $<0.001$ & 1.628 & 3.601 \\
\hline
\end{tabular}

This analysis is controlled Insomnia Severity Index. Bootstrap sample size: 5000, LL: lower limit, UL: upper limit, CI: bias corrected 95\% bootstrap confidence interval. 


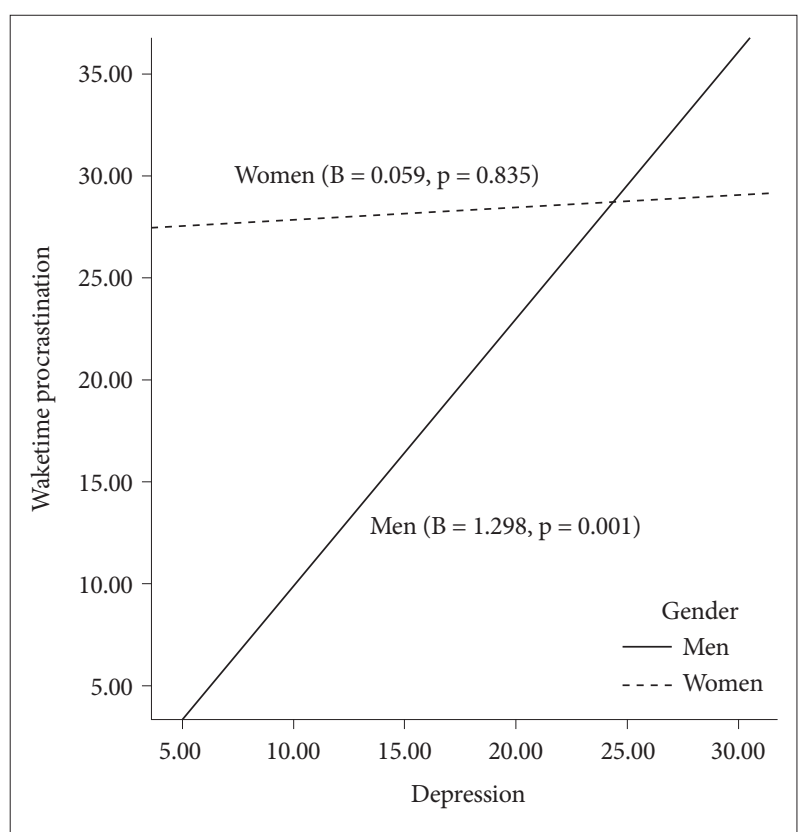

Fig. 1. Gender differences in waketime procrastination on weekdays.

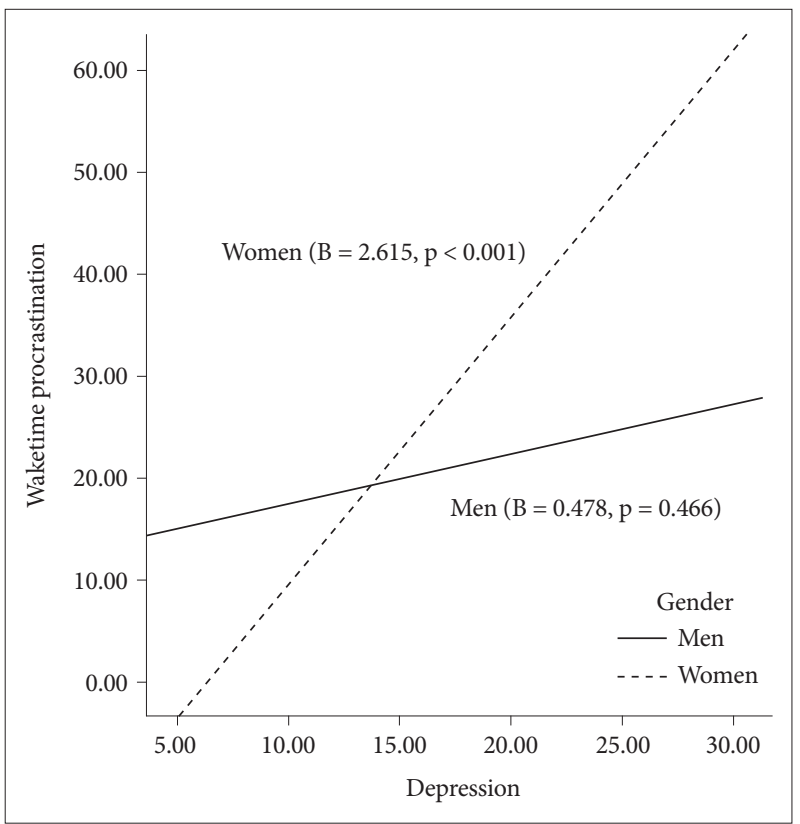

Fig. 2. Gender differences in waketime procrastination on weekends.

gaged in more WP than their non-depressed counterparts. Women reported more WP than men on both weekdays and weekends. When men did engage in WP, they reported more WP on weekends than weekdays. Only men with high depression showed significantly more WP on weekdays, while only women with high depression showed significantly more WP on weekends. To the best of our knowledge, this is the first study investigating WP in the context of insomnia and depression considering gender differences.

\section{Waketime Procrastination and Depression}

Our study found that higher levels of depression was associated with higher levels of WP in insomnia patients; this was consistent with previous studies reporting that depressed people spend prolonged TIB [5-7,20,21]. WP may be a behavior that indicates low motivation levels among major depression disorder patients. Depressed people often complain of reduced energy levels and feelings of helplessness, which often makes it difficult to get ready for work in the morning $[6,7,21,22]$. Low levels of motivation in depressed people can also be found in neuroimaging studies. People with depression have decreased serotonin in the ventromedial prefrontal cortex, and dopamine deficiency is observed due to abnormal activity in the ventral striatum, which has been associated with procrastination, and lack of motivation $[23,24]$. Therefore, low mood and lack of motivation, especially in the morning, may cause depressed people to have difficulty getting out of bed in the morning.

It is well known that patients with severe depression feel depressed in the mornings and gradually improve as the day progresses [25]. According to Lemke's study [26], patients with depression reported significantly higher levels of depression in the morning. Healthy people experienced a decrease in depression after getting sufficient sleep, but sleep disturbance may exacerbate depression symptoms. This is thought to be caused by qualitative differences in sleep patterns of depressed people. Therefore, depressed people can feel more depressed in the morning after sleeping [27]. In fact, depressed patients have lower blood thyroxin levels in the morning, which is associated with a depressed mood. According to one study, patients with depression had lower levels of Vanilmandelate and Homovalilate in their urine in the morning compared to in the evening, which was related to lower thyroxin levels [28].

Additionally, depressed people may want to stay in bed longer in the morning to recover from fatigue. Depressed people may feel more subjective fatigue in the morning than less-depressed people because their morning cortisol levels are higher than those who are less depressed [26,29].

\section{The Moderating Effect of Gender in the Relationship between WP and Depression on Weekdays and Weekends}

In this study, although gender differences for WT was not significant, women had more WP than men, which may be due to gender differences in depression that are reflected in WP. Gender differences in depression are widely known, and women generally report a higher level of depression than men [30]. In this study, gender moderated the relationship between depression and WP. In our study, depressed men reported more WP on weekdays than women, which suggests that depression in men may mainly be a reflection of social withdrawal [31]. Generally, there are social schedules such as work and school during weekdays, and the severity of depression in men may lead to prolonged 
WP in order to delay starting these social schedules during the weekdays. Meanwhile, the same effect was not found on weekends for men, which may be related to the characteristics of coping styles for depression more likely in men. Studies have reported that men are more likely to choose active coping strategies to alleviate a depressed mood compared to women [14]. This suggests that on weekends when there are limited social schedules, depressed men are likely to get out of bed in order to actively cope with their depressed mood.

Women reported more WP on weekends, but the effect was not significant during weekdays. Rumination is a major predictor of depression, and women use rumination more often than men to cope with depression $[9,32]$. Depressed people tend to use the bed as escape to cope with their depression, and from a behavioral perspective, rumination helps depressed individuals avoid the aversive environment surrounding them $[7,9]$. Also, depressed women tend to complain more about fatigue and physical pain than depressed men $[33,34]$.

This is the first published study to explore gender differences in the relationship between WP and depression. While it is widely known that staying in bed for prolonged periods of time is associated with depression and insomnia, previous sleep studies have focused on pre-sleeping behavior (such as bedtime procrastination) and have overlooked WP. In this study, we investigated gender differences in the relationship between WP and depression and suggest the need for interventions for WP in the sleep treatment of insomniac patients with depression.

The relationship between depression and WP may differ based on gender and weekdays and weekends differences. For example, individuals are generally more stressed due to their job during weekdays than on the weekend, and this stress has been identified as a trigger for depressive symptoms in men [35]. Based on the literature, psychosocial factors such as financial and professional failure are especially high-risk factors for depression among men [30]. This suggests that they may experience more depressive symptoms than women during the weekdays, thus depression in men may be reflected by greater WP during weekdays than women. In addition, there is more opportunity for individuals to cope with their emotions on the weekends [36]. In coping with depression, women prefer less active solutions than men [14], indicating that depressed women may exhibit higher WP than depressed men on the weekends.

In our study, both men and women reported more WP on weekends than weekdays; this is consistent with the hypothesis of this study that WP is more likely to occur on weekends when there are less required social schedules. In general, people lie in bed longer on weekends than on weekdays [37], and previous studies have reported that deviations in sleep patterns during weekends compared to weekdays have a negative effect on sleep and mood [38]. Extending this view, our study suggests that weekday-weekend differences in WP may also negatively affect an individual's sleep and emotions.

\section{Clinical Implications}

In general, cognitive behavior therapy for insomnia uses behavioral techniques such as sleep restriction and stimulation control, but often does not directly assess or provide interventions specifically targeting WP. In this study, WP was measured using a 7-day sleep diary utilizing the difference between WT and time out of bed. This is a sleep parameter that is not often used to guide treatment in most behavioral interventions for insomnia or depressed individuals. Our study found gender differences in WP, which is generally consistent with where women report more insomnia, and emphasized the importance of therapeutic interventions tailored to gender [11]. Insomnia patients who have long WP should also be screened for depression. Also, clinicians can check their lifestyle or activities and recommend the better coping strategies based on gender.

\section{Limitation}

This study was an exploratory study of gender differences in the relationship between WP and depression in a clinical group with insomnia. This study relies on subjective reports of sleep and depression. Further studies using objective measures of sleep (e.g., polysomnography and actigraphy) and biological indicators of depression (e.g., fMRI) may provide more information as to why depression is associated with high levels of WP. Second, the current study was a cross-sectional study investigating WP. These observations suggest that WP associated with insomnia and depression may be more pronounced in women. In future research, it is necessary to explore psychological mechanisms of WP through longitudinal studies. Third, this study did not collect information about employment, years of education, marital status, parental status, and cohabitation, which may have been helpful in identifying the population. Fourth, since this study did not examine coping styles, there may be limitations in interpreting the effects of gender. Finally, this study is limited to a relatively young age group (20-40 age range). Age has been shown to be significantly associated with a bigger discrepancy between weekday and weekends due to stronger eveningness tendency $[39,40]$. Thus, future studies may need to replicate the relationship between WP and depression in an older age group.

\section{Conclusion}

This study investigated gender differences in the relationship between WP and depression in an insomniac group. High levels of depression were associated with high levels of WP. Men with high depression showed more WP on weekdays and women with high depression showed more WP on weekends. WP is often overlooked in sleep studies and treatment; our study suggests that WP is an important behavior to consider in the clinical context of providing sleep therapy to depressed individuals, particularly while taking gender differences into account. 


\section{Acknowledgments}

This work was supported by the National Research Foundation of Korea (NRF) grant funded by the Korea government (MSIT) (NRF-2017R1C1B 1008002).

\section{Conflicts of Interest}

The authors have no financial conflicts of interest.

\section{Authors' Contribution}

Conceptualization: Kim J, Suh S. Data curation: Kim J, Suh S. Formal analysis: Kim J, Suh S. Methodology: Kim J, Suh S. Project administration: Suh S. Supervision: Suh S. Visualization: Kim J, Suh S. Writing-original draft: Kim J, Suh S. Writing-review \& editing: Suh S.

\section{REFERENCES}

1. Natale V, Léger D, Martoni M, Bayon V, Erbacci A. The role of actigraphy in the assessment of primary insomnia: a retrospective study. Sleep Med 2014;15:111-5.

2. Bos SC, Macedo AF. Literature review on Insomnia (2010-2016). Biol Rhythm Res 2019;50:64-163.

3. Sunderajan P, Gaynes BN, Wisniewski SR, Miyahara S, Fava M, Akingbala $\mathrm{F}$, et al. Insomnia in patients with depression: a STAR*D report. CNS Spectr 2010;15:394-404.

4. Franzen PL, Buysse DJ. Sleep disturbances and depression: risk relationships for subsequent depression and therapeutic implications. Dialogues Clin Neurosci 2008;10:473-81.

5. Manber R, Bernert RA, Suh S, Nowakowski S, Siebern AT, Ong JC. CBT for insomnia in patients with high and low depressive symptom severity: adherence and clinical outcomes. J Clin Sleep Med 2011;7:645-52.

6. Wells KB, Stewart A, Hays RD, Burnam MA, Rogers W, Daniels M, et al. The functioning and well-being of depressed patients. Results from the Medical Outcomes Study. JAMA 1989;262:914-9.

7. Kanter JW, Baruch DE, Gaynor ST. Acceptance and commitment therapy and behavioral activation for the treatment of depression: description and comparison. Behav Anal 2006;29:161-85.

8. Sivertsen B, Vedaa Ø, Harvey AG, Glozier N, Pallesen S, Aarø LE, et al. Sleep patterns and insomnia in young adults: a national survey of Norwegian university students. J Sleep Res 2019;28:e12790.

9. Girgus J, Yang K. Gender and depression. Curr Opin Psychol 2015;4:5360.

10. Van de Velde S, Bracke P, Levecque K. Gender differences in depression in 23 European countries. Cross-national variation in the gender gap in depression. Soc Sci Med 2010;71:305-13.

11. Suh S, Cho N, Zhang J. Sex differences in insomnia: from epidemiology and etiology to intervention. Curr Psychiatry Rep 2018;20:69.

12. Mong JA, Cusmano DM. Sex differences in sleep: impact of biological sex and sex steroids. Philos Trans R Soc Lond B Biol Sci 2016;371:20150110.

13. Woosley JA, Lichstein KL, Taylor DJ, Riedel BW, Bush AJ. Predictors of perceived sleep quality among men and women with insomnia. Behav Sleep Med 2012;10:191-201.

14. Piccinelli M, Wilkinson G. Gender differences in depression. Critical review. Br J Psychiatry 2000;177:486-92.

15. Radloff LS. The CES-D scale: a self-report depression scale for research in the general population. Appl Psychol Meas 1977;1:385-401.

16. Lee S, Oh ST, Ryu SY, Jun JY, Lee K, Lee E, et al. Validation of the Korean version of Center for Epidemiologic Studies Depression ScaleRevised (K-CESD-R). Korean J Psychosom Med 2016;24:83-93.

17. Cho YW, Song ML, Morin CM. Validation of a Korean version of the insomnia severity index. J Clin Neurol 2014;10:210-5.

18. Van Dam NT, Earleywine M. Validation of the Center for Epidemiologic Studies Depression Scale--Revised (CESD-R): pragmatic depres- sion assessment in the general population. Psychiatry Res 2011;186:12832 .

19. Shrout PE, Bolger N. Mediation in experimental and nonexperimental studies: new procedures and recommendations. Psychol Methods 2002; 7:422-45.

20. Murray CB, Murphy LK, Palermo TM, Clarke GM. Pain and sleep-wake disturbances in adolescents with depressive disorders. J Clin Child Adolesc Psychol 2012;41:482-90.

21. Jacobson NS, Martell CR, Dimidjian S. Behavioral activation treatment for depression: returning to contextual roots. Clin Psychol Sci Pract 2001; 8:255-70.

22. Nolen-Hoeksema S, Wisco BE, Lyubomirsky S. Rethinking rumination. Perspect Psychol Sci 2008;3:400-24.

23. Koenigs M, Grafman J. The functional neuroanatomy of depression: distinct roles for ventromedial and dorsolateral prefrontal cortex. Behav Brain Res 2009;201:239-43.

24. Nestler EJ, Carlezon WA Jr. The mesolimbic dopamine reward circuit in depression. Biol Psychiatry 2006;59:1151-9.

25. Nutt D, Wilson S, Paterson L. Sleep disorders as core symptoms of depression. Dialogues Clin Neurosci 2008;10:329-36.

26. Lemke MR, Broderick A, Zeitelberger M, Hartmann W. Motor activity and daily variation of symptom intensity in depressed patients. Neuropsychobiology 1997;36:57-61.

27. Cartwright R, Baehr E, Kirkby J, Pandi-Perumal SR, Kabat J. REM sleep reduction, mood regulation and remission in untreated depression. Psychiatry Res 2003;121:159-67.

28. Birkmayer W, Riederer P. Biochemical post-mortem findings in depressed patients. J Neural Transm 1975;37:95-109.

29. Knorr U, Vinberg M, Kessing LV, Wetterslev J. Salivary cortisol in depressed patients versus control persons: a systematic review and metaanalysis. Psychoneuroendocrinology 2010;35:1275-86.

30. Kendler KS, Gardner CO. Sex differences in the pathways to major depression: a study of opposite-sex twin pairs. Am J Psychiatry 2014;171: 426-35.

31. Funabiki D, Bologna NC, Pepping M, FitzGerald KC. Revisiting sex differences in the expression of depression. J Abnorm Psychol 1980;89: 194-202.

32. Johnson DP, Whisman MA. Gender differences in rumination: a metaanalysis. Pers Individ Dif 2013;55:367-74.

33. George MS, Ketter TA, Parekh PI, Herscovitch P, Post RM. Gender differences in regional cerebral blood flow during transient self-induced sadness or happiness. Biol Psychiatry 1996;40:859-71.

34. Munce SE, Stewart DE. Gender differences in depression and chronic pain conditions in a national epidemiologic survey. Psychosomatics 2007; 48:394-9.

35. Schlotz W, Hellhammer J, Schulz P, Stone AA. Perceived work overload and chronic worrying predict weekend-weekday differences in the cortisol awakening response. Psychosom Med 2004;66:207-14.

36. Ryan RM, Bernstein JH, Brown KW. Weekends, work, and well-being: psychological need satisfactions and day of the week effects on mood, vitality, and physical symptoms. J Soc Clin Psychol 2010;29:95-122.

37. Monk TH, Buysse DJ, Rose LR, Hall JA, Kupfer DJ. The sleep of healthy people--a diary study. Chronobiol Int 2000;17:49-60.

38. Lund HG, Reider BD, Whiting AB, Prichard JR. Sleep patterns and predictors of disturbed sleep in a large population of college students. J Adolesc Health 2010;46:124-32.

39. Fernández-Mendoza J, Ilioudi C, Montes MI, Olavarrieta-Bernardino S, Auirre-berrocal A, De la Cruz-Troca JJ, et al. Circadian preference, nighttime sleep and daytime functioning in young adulthood. Sleep Biol Rhythms 2010;8:52-62.

40. Fischer D, Lombardi DA, Marucci-Wellman H, Roenneberg T. Chronotypes in the US-Influence of age and sex. PLoS One 2017;12:e0178782. 